\title{
Dynamics of a nonlinear dipole vortex
}

J. S. Hesthaven, J. P. Lynov, A. H. Nielsen, J. Juul Rasmussen, and M. R. Schmidt

Association EURATOM-Risó National Laboratory, Optics and Fluid Dynamics Department, P. O. Box 49,

DK-4000 Roskilde, Denmark

E. G. Shapiro and S. K. Turitsyn

Institute of Automation and Electrometry, Russian Academy of Sciences, Novosibirsk 630090, Russia

(Received 29 June 1994; accepted 23 May 1995)

A localized stationary dipole solution to the Euler equations with a relationship between the vorticity and streamfunction given as $\omega=-\psi+\psi^{3}$ is presented. By numerical integration of the Euler equations this dipole is shown to be unstable. However, the initially unstable dipole reorganizes itself into a new nonlinear dipole, which is found to be stable. This new structure has a functional relationship given as $\omega=\alpha \psi+\beta \psi^{3}-\gamma \psi^{5}$. Such dipoles are stable to head-on collisions and they are capable of creating tripolar structures when colliding off axis. The effects of increasing Newtonian viscosity on the nonlinear dipole is studied revealing that even though the nonlinearity is weakening, the dipole does not relax towards a Lamb dipole. (C) 1995 American Institute of Physics.

\section{INTRODUCTION}

The dipole vortex is one of the fundamental vortical structures of two-dimensional flows. The dipole consists essentially of two closely packed regions of opposite vorticity (i.e., two monopole vortices). Often the dipole contains a net linear momentum, which makes it translate, in contrast to the monopole, which only contains angular momentum. However, dipolar solutions which are not propagating may also exist, as will be discussed in the following section. We term these structures shielded dipoles. Propagating dipolar vortices are easily excited in two-dimensional flows and they appear to be the universal outcome of an external forcing possessing a nonzero linear momentum. They have for example been excited by a pulsed jet in both stratified, ${ }^{1-3}$ rotating ${ }^{4-6}$ and conducting fluids controlled by an external magnetic field, ${ }^{7}$ by moving a thin cylinder through a soap film, ${ }^{8}$ or moving a vertical cylindrical tube through a rotating fluid. ${ }^{9}$ They have further been formed in rapidly rotating fluids in a parabolic vessel. ${ }^{10}$ Two dipoles have also been found to appear from an unstable anticyclonic vortex in a rotating fluid ${ }^{11}$ and, finally, dipoles have been observed to appear spontaneously in high resolution numerical investigations of forced two-dimensional turbulence. ${ }^{12}$

Dipolar vortex structures are stationary solutions to the Euler equations describing two-dimensional, inviscid flows. These solutions are characterized by a functional relationship between the vorticity, $\omega$, and the streamfunction, $\psi$, i.e., $\omega=f(\psi)$, where $f$ is an arbitrary integrable function. For the simplest case, where $f$ is a linear function, one finds the well-known Lamb dipole, which was derived by Lamb. ${ }^{13}$ According to a recent paper, ${ }^{14}$ a similar structure, in addition to more general dipole solutions with linear $f$, was derived independently by Chaplygin around the turn of the century. This solution has a rotational interior separated from an irrotational exterior by a circular separatrix. Although observed dipoles often are well described by the Lamb dipole, there are several examples, where the function $f$ appears to be nonlinear. This was for instance found for the dipoles excited by a turbulent jet in a stratified flow ${ }^{3}$ and also for the dipoles investigated by Nguyen Duc and Sommeria ${ }^{7}$ in a conducting fluid. The form of $f$ in these cases appear to be close to a sinh function [i.e., $\omega=A \sinh (c \psi)$ ]. A similar relationship was also observed in numerical simulations were the dipole was emerging from two nearby Gaussian monopoles of opposite signed vorticity ${ }^{8,15}$ and for the reconstructed dipoles appearing after off-axis collision between two Lamb dipoles. ${ }^{16}$ The $\sinh$ relationship was predicted several years ago by Joyce and Montgomery, ${ }_{17}$ using a statistical mechanics approach, as characterizing the most probable state of a two-dimensional system of ideal point vortices. Recent, long time, high resolution, double periodic numerical calculations have verified that the "final" relaxed stationary state of twodimensional flows obey this relationship. ${ }^{18,19}$ Other similar approaches also based on statistical mechanical theories, where the characteristic relaxed states are found by maximizing the entropy, have revealed relationships close to the sinh relationship ${ }^{20,21}$ for the most probable state of self-organized two-dimensional flows and could, under certain conditions, reproduce the sinh relationship; see, e.g.; the discussion in the recent work of Pasmanter. ${ }^{22}$

On the basis of these investigations, one might expect that the sinh-type dipoles, together with the Lamb dipole, are the most relevant ones among the infinite class of possible stationary solutions. One should point out, however, that the application of the above-mentioned theories to describe the formation of a dipole from a spatially localized perturbation in an unbounded fluid is delicate. The dipolar structure will probably appear as a local entropy maximum, but cannot be a global maximum.

We have investigated a dipolar structure with a different nonlinear functional relationship. For a particular nonlinear form of $f, f(\psi)=-\psi+\psi^{3}$, a localized solution with a dipolar structure has been found. This solution, which is derived in Sec. II, is characterized by having two cores of opposite vorticity, each of which is shielded by a region with vorticity of opposite sign and the structure is not moving. We term it a shielded dipole. The stability of this solution is investigated by solving the Euler equations numerically with the solution as the initial condition. The shielded dipole solution is ob- 
served to be unstable. It splits into two propagating dipoles moving in opposite directions: a "strong" dipole, composed of the vorticity in the two cores and a "weak" dipole composed of the vorticity initially shielding the two cores. The "strong" dipole is found to be steadily propagating. From scatterplots, we confirmed that this dipole reaches a stationary state with a nonlinear relationship between $\omega$ and $\phi$, where $\phi$ is the streamfunction in the frame moving with the dipole, the "co-moving" streamfunction. We find that $f(\phi)$ is well approximated by $f(\phi) \approx \alpha \phi+\beta \phi^{3}-\gamma \phi^{5}$ (with the constants $\alpha, \beta$, and $\gamma$ positive) inside a separatrix of elliptical shape, elongated perpendicular to the direction of propagation, and $f(\phi)=0$ outside the separatrix. This appears to be a new type of stationary dipole solution to the twodimensional Euler equations. We refer to this as a nonlinear dipole. We have further investigated the nonlinear dynamics of this dipole numerically, and found that the nonlinear dipole is stable to head-on collisions, as is the Lamb dipole. We have also revealed that an off-axis collision between two nonlinear dipoles may lead to the formation of a tripolar structure as also found for Lamb dipoles. Making the nonlinear dipole collide head-on with a Lamb dipole we found that both types of dipoles regenerated after the interaction and continued to propagate along their original direction away from each other. Finally, we investigated the influence of Newtonian viscosity on the evolution of the nonlinear dipole. It was found to damp, while the vorticity was spreading out, however, it kept a nonlinear relationship between $\omega$ and $\phi$.

The paper is organized as follows: In the following section we derive the shielded localized dipolar structure, and we briefly discuss the relevance of this solution to other fields. In Sec. III, we describe the numerical investigations of the stability of the shielded dipole and the dynamics of the nonlinear dipole. We compare the functional relationship between $\omega$ and $\phi$ for the steadily propagating nonlinear dipole with the predictions from the mean-field statistical theories mentioned above. Finally, Sec. IV contains our conclusions.

\section{SHIELDED DIPOLE SOLUTION TO THE EULER EQUATIONS}

Consider the two-dimensional Euler equations in the vorticity streamfunction formulation

$$
\begin{aligned}
& \frac{\partial \omega}{\partial t}+[\omega, \psi]=0, \\
& \nabla^{2} \psi=-\omega,
\end{aligned}
$$

where $(x, y) \in \Omega, \psi: \Omega \rightarrow R$ is the streamfunction, $\omega: \Omega \rightarrow R$ is the vorticity and $[\cdot, \cdot]$ designates the Jacobian. The velocity field is related to the streamfunction as

$$
u=\frac{\partial \psi}{\partial y}, \quad v=-\frac{\partial \psi}{\partial x} .
$$

From Eq. (1) we observe that an infinite class of stationary solutions exists for which

$$
\omega=f(\psi)
$$

where $f(\psi)$ is an arbitrary, integrable function of $\psi$. Note, that $f$ should be different from a constant in order to obtain nontrivial solutions.

A well-known and particularly simple example of a localized stationary solution to the Euler equations is the Lamb dipole, ${ }^{13,14}$ where $f(\psi)=\lambda^{2} \psi$ inside a circle and $f(\psi)=0$ outside; $\lambda$ is a constant. Thus the dipole has a localized rotational interior and an irrotational exterior, with the two domains separated by a circular separatrix. For this choice of $f$, Eq. (3) may be solved analytically, and the solution obtained in a closed form in terms of first-order Bessel functions of the first kind. We should note that this solution has discontinuous odd derivatives of the vorticity $\omega$ at the separatrix.

In the present paper, we are aiming at finding a localized stationary solution with

$$
\omega=f(\psi)=-\psi+\psi^{3},
$$

or likewise

$$
\psi_{x x}+\psi_{y y}-\psi+\psi^{3}=0
$$

where subscripts designate differentiation. Solutions to Eq. (5) may, besides being studied in the context of the Euler equations, also be applied as two-dimensional stationary solutions to the nonlinear Schrödinger equation, which is one of the most applied and well-studied equations modeling a variety of wave phenomena, e.g., self-focusing of light beams, propagation of high-frequency plasma waves, water waves, etc. (see, e.g., Ablowitz and Segur ${ }^{23}$ ). Solutions to Eq. (5) may also be interpreted as stationary solutions to the Ginzburg-Landau equations or the nonlinear heat equation under some assumptions. Hence, the solutions found for Eq. (5) are of general interest. Solutions to Eq. (5) with radial symmetry are well studied. ${ }^{24,25}$ Also, a solution consisting of five vortices has recently been derived. ${ }^{26}$

Here, we present a solution with dipolar structure, i.e., one with a nontrivial symmetry. ${ }^{27}$ Thus we search for solutions with the following symmetries:

$$
\psi(x, y)=-\psi(-x, y), \quad \psi(x, y)=\psi(x,-y),
$$

i.e., a solution which is even in $y$ and odd in $x$. The symmetries allow for solving Eq. (5) in the first quadrant $(x \geqslant 0, y \geqslant 0)$ only. The restrictions posed by Eq. (6) imply the following boundary conditions on the axes:

$$
\psi(0, y)=0,\left.\quad \frac{\partial \psi(x, y)}{\partial y}\right|_{y=0}=0 .
$$

Additionally, we may use the fact that the objective of our interest is localized structures for which $\psi$ vanishes as $r$ $=\sqrt{x^{2}+y^{2}} \rightarrow \infty$. Hence, when $r$ goes towards infinity, the influence of the nonlinear term in Eq. (5) is negligible and $\psi$ will have the following asymptotic behavior:

$$
\psi(r, \theta)=A K_{1}(r) \cos \theta \simeq A \sqrt{\frac{\pi}{2 r}} \exp (-r) \cos \theta,
$$

where $K_{1}$ is the first-order modified Bessel function of the first kind, $A$ is a constant amplitude, and $\tan \theta=y / x$. Choosing higher-order azimuthal modes and changing the boundary conditions accordingly leads to more exotic topologies, 
e.g., quadrupoles, etc. In the numerical solution of Eq. (5), we have used Eq. (8) as the asymptotic boundary condition on $\psi$.

Using Hamilton's principle we may reformulate Eq. (5) as

$$
\delta S=\delta \int_{\Omega} L\left(x, y, \psi, \psi_{x}, \psi_{y}\right) d x d y=0,
$$

where the Lagrangian has the form

$$
L\left(x, y, \psi, \psi_{x}, \psi_{y}\right)=\frac{\psi_{x}^{2}}{2}+\frac{\psi_{y}^{2}}{2}+\frac{\psi^{2}}{2}-\frac{\psi^{4}}{4} .
$$

This may easily be verified by inserting the Lagrangian into the Euler-Lagrange equation

$$
\frac{\partial L}{\partial \psi}-\frac{\partial}{\partial x} \frac{\partial L}{\partial \psi_{x}}-\frac{\partial}{\partial y} \frac{\partial L}{\partial \psi_{y}}=0,
$$

to obtain Eq. (5). Equation (9) allows for deriving some integral relations, which are useful for the numerical solution of Eq. (5). For localized solutions, one may find these directly from the variational principle, since the solution, $\psi$, of Eq. (5) is a maximum of the functional $S$ and a small variation of $S$ around $\psi$ must equal zero. Choosing the trial function as $f=\alpha \psi(x, y)$ yields the following identity:

$$
\left.\frac{d S[f]}{d \alpha}\right|_{\alpha=1}=0 .
$$

Applying Hamilton's principle to this, immediately gives the relation

$$
\int_{\Omega}\left(\psi_{x}^{2}+\psi_{y}^{2}\right) d x d y+\int_{\Omega} \psi^{2} d x d y=\int_{\Omega} \psi^{4} d x d y .
$$

Likewise, we may choose the trial functions $g=\psi(\beta x, y)$ and $h=\psi(x, \gamma y)$. Introducing these functions into the functional $S$ yields, for small variations of $\beta$ and $\gamma$ around 1 , the equations

$$
\left.\frac{d s[g]}{d \beta}\right|_{\beta=1}=0,\left.\quad \frac{d s[h]}{d \gamma}\right|_{\gamma=1}=0 .
$$

Applying Hamilton's principle on these implies, after some algebra, the additional integral equalities

$$
\begin{aligned}
& \int_{\Omega} \psi_{y}^{2} d x d y+\int_{\Omega} \psi^{2} d x d y \\
& =\int_{\Omega} \psi_{x}^{2} d x d y+\frac{1}{2} \int_{\Omega} \psi^{4} d x d y, \\
& \int_{\Omega} \psi_{x}^{2} d x d y+\int_{\Omega} \psi^{2} d x d y \\
& =\int_{\Omega} \psi_{y}^{2} d x d y+\frac{1}{2} \int_{\Omega} \psi^{4} d x d y .
\end{aligned}
$$

Also, we may derive some simple expressions for the energy, $E$, and the enstrophy, $W$, by manipulations of the integral constraints:

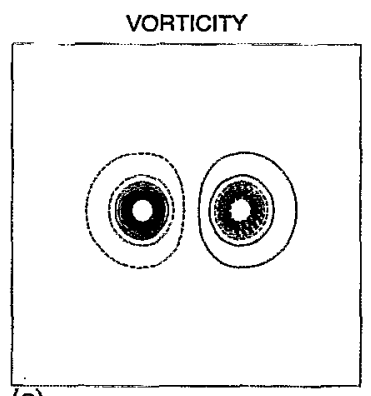

STREAMFUNCTION

(a)

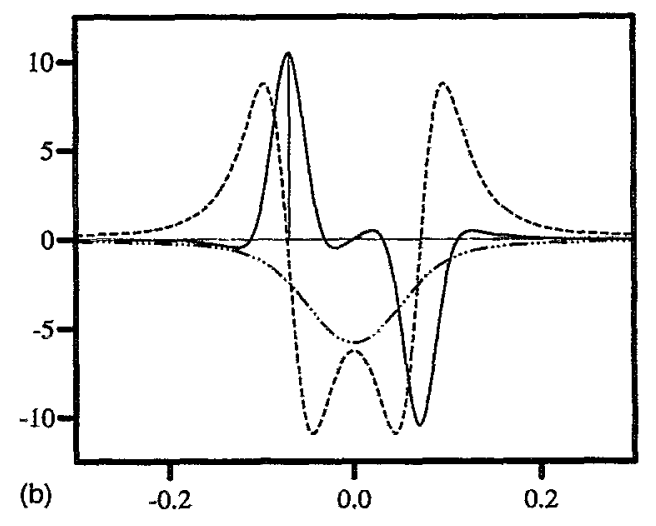

FIG. 1. The nonlinear dipole solution to Eq. (5). (a) Contour plots of the streamfunction, $\psi$, and of the vorticity, $\omega$. Full lines indicate positive values while dashed lines indicate negative values. (b) The variation of the vorticity along the $x$ axis (full line) and of the $y$ component of the velocity field along the $x$ axis (dashed line) and $y$ axis (dashed-dotted line).

$$
\begin{aligned}
& E=\frac{1}{2} \int_{\Omega}(\nabla \psi)^{2} d x d y=\frac{1}{2} \int_{\Omega} \psi^{2} d x d y \\
& W=\frac{1}{2} \int_{\Omega} \omega^{2} d x d y=\frac{1}{2} \int_{\Omega}\left(\psi_{x x}^{2}+\psi_{y y}^{2}-2 \psi^{2}\right) d x d y
\end{aligned}
$$

Employing the boundary conditions Eqs. (7) and (8) and the integral constraints Eqs. (13)-(16) we solve Eq. (5) numerically by a two-dimensional shooting method. Figure 1(a) displays the found solution, $\psi$. As expected, it is a clearly localized dipolar structure, with two opposite signed poles. The corresponding vorticity distribution is also illustrated. Here we note that the cores of the structure are surrounded by regions of opposite signed vorticity. Hence, the structure is shielded. This is also illustrated in Fig. 1(b), where we have plotted the variation of the vorticity along the $x$ axis and the variation of the $y$ component of the velocity along both the $x$ axis and the $y$ axis. It is observed that the velocity at each of the vorticity extrema of the two poles is vanishing and the structure is not moving. There is no separatrix for this solution and it is continuous in all higher derivatives. As far as we know, this new solution is the first example of a nonlinear localized dipole, where the localization appears naturally. However, due to the shielding we may expect that the structure is unstable, since it is well known that shielded monopolar vortices may be unstable to azimuthal perturbations, ${ }^{28}$ 

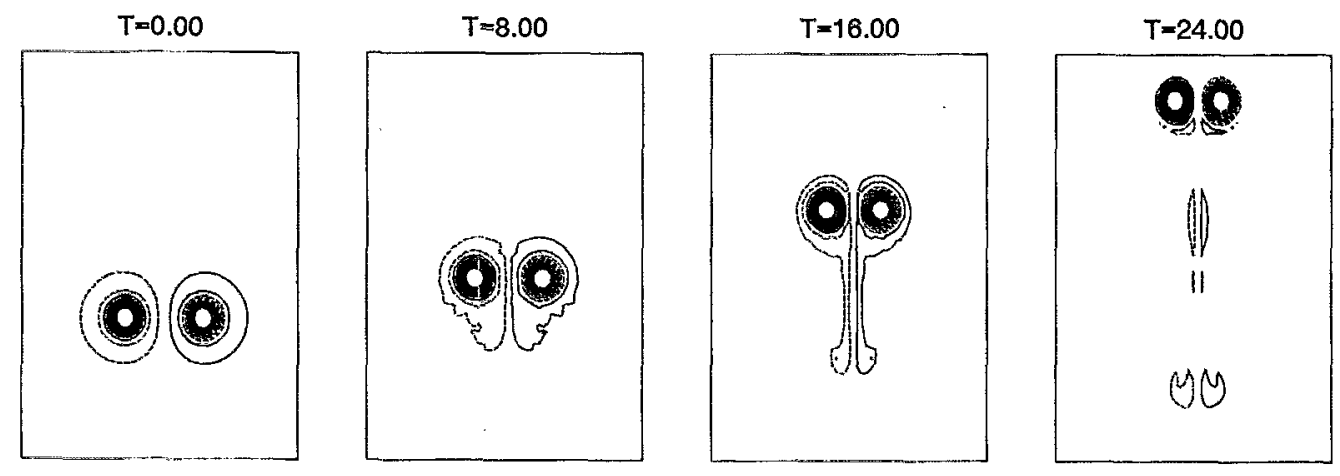

FIG. 2. Contour plots of the temporal evolution of the vorticity of the time-integrated Euler equations with the nonlinear dipole as initial condition. The spectral resolution is here $N_{x}=128$ and $N_{y}=512$.

and for the present structure each of the poles introduces a strong perturbation on the other. Also, it was recently found that a shielded Lamb dipole is unstable. ${ }^{29}$

\section{DYNAMICS OF THE NONLINEAR DIPOLE SOLUTION}

In order to study the stability and dynamics of the dipole solution described in the previous section, we have solved the Euler equations Eq. (1) numerically using a standard double periodic, fully dealiased spectral code. In the code a solution to Eq. (1) is expanded in a Fourier series:

$$
\begin{aligned}
\omega(x, y)= & \sum_{k_{x}=-N_{x} / 2}^{N_{x} / 2} \sum_{k_{y}=-N_{y} / 2}^{N_{y} / 2} \omega_{k_{x} k_{y}} \\
& \times \exp \left(\frac{2 \pi i k_{x} x}{l_{x}}\right) \exp \left(\frac{2 \pi i k_{y} y}{l_{y}}\right),
\end{aligned}
$$

where $l_{x}$ and $l_{y}$ are the size of our computational domain and $N_{x}$ and $N_{y}$ are the spectral resolution. Note that although we have the possibility of having $l_{x} \neq l_{y}$, we always choose $N_{x} / N_{y}=l_{x} / l_{y}$. The time integration is performed by a fully corrected Adams-Bashforth third-order scheme. For further details on the code see, e.g., Coutsias et al. ${ }^{30}$

The algorithm described above leads to a stable scheme provided the dynamics of the Euler equations is well resolved. However, for cases where the spectral cascade to smaller scales become significant, e.g., in off-axis collisions between dipoles, the appearance of Gibb's phenomenon may render the scheme unstable. To ensure numerical stability of the scheme, also in these cases, we apply a high-order filter of the form $\mu \nabla^{6} \omega$, which is implemented by a backward Euler scheme. The filter has the effect of allowing for reconnection of isolines of the vorticity on small scales. Since we wish to study the dynamics of the Euler equations, it is beneficial to keep the effects of dissipation at a minimum, which is most conveniently done by applying higher order filtering as opposed to Newtonian viscosity. For a typical case with $\mu=10^{-7}$ we found the energy loss due to the filtering to be less than $1 \%$, while the loss of enstrophy was less than $10 \%$. The effects of significant Newtonian viscosity, represented by the term $\nu \nabla^{2} \omega$, on the dynamics of the structures will be addressed in Sec. III C. Also, here the viscous term is handled by a backward Euler scheme.

\section{A. Stability of the shielded dipole}

In Fig. 2 we display the result of a time integration of the Euler equations with the shielded dipole, derived in Sec. II and depicted in Fig. 1, as the initial condition. We have here used a resolution of 128 modes in the $x$ direction and 512 modes in the $y$ direction. With $\mu=10^{-7}$, the energy loss is $0.5 \%$ during the simulation and the enstrophy loss is $5.0 \%$, where the energy and enstrophy are defined in Eqs. (17) and (18). The loss of enstrophy is much larger than the energy loss since enstrophy is concentrated at the smaller scales as compared to the energy. In Fig. 2 we observe that the shielded dipole is unstable and breaks up during a few turnover times in the central part of each of the poles (one turnover period, defined as $2 \pi / \omega$, where $\omega$ is taken at the position of maximum azimuthal velocity, corresponds to around 1.2 in our normalized units). However, we observe that the core of the nonlinear dipole survives, and reorganizes into a new, strongly localized dipole structure. Whereas the initial dipole was fixed in space, this product dipole, having left behind the residual shielding, propagates with constant velocity. A significant difference between our product dipole and the Lamb dipole is that for the former the two centers are more separated and therefore the speed is comparatively slower. However, the centers of the two poles in the moving product dipole are somewhat closer than the corresponding centers of the initial shielded dipole.

To study the topological changes occurring during the breakup and reorganization phase, we show in Fig. 3 a time sequence of the scatterplots where the vorticity, $\omega$, is plotted against the "comoving" streamfunction, $\phi=\psi+U(T) x$. Here $U(T)$ is the velocity of the structure with $U(T=0)=0$. At $T=0$, we see the clear third-order polynomial relationship between the vorticity and the streamfunction. We also observe that the dipole is naturally localized, i.e., there is no separatrix separating a rotational and an irrotational region. The instability of the initial shielded dipole causes the dipole to break up, and we see that the reorganization of the strong 

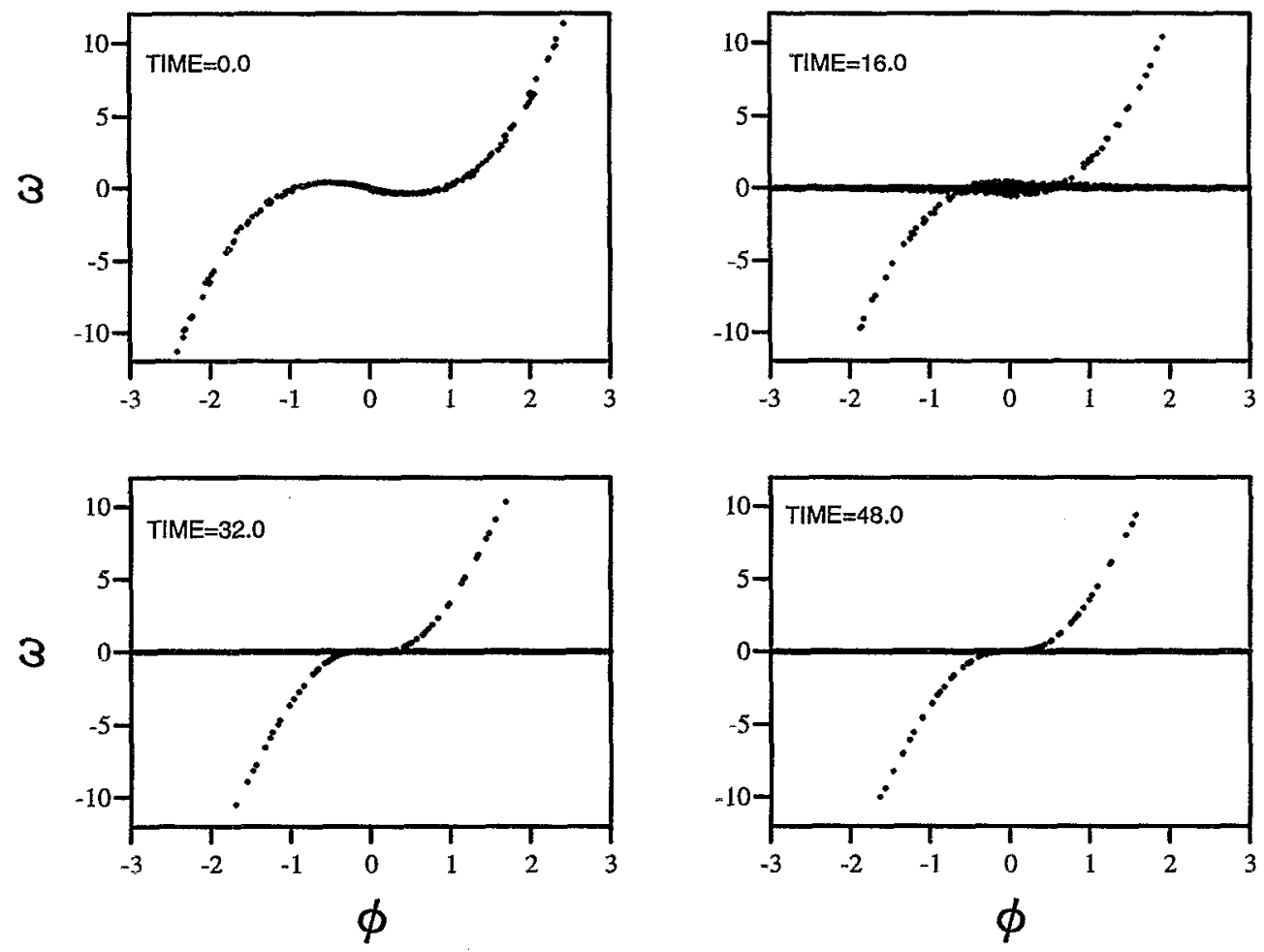

FIG. 3. Time sequence of scatterplots for the nonstationary reorganization of the nonlinear dipole into the product dipole. $\phi=\psi+U x$ designates the streamfunction in the frame moving with the dipole.

product dipole, which we shall refer to as the nonlinear dipole, implies the appearance of a separatrix as this structure starts to move. (Note that the scatterplots are obtained only over the region containing the nonlinear dipole.) The separatrix is found to have an elliptical shape elongated in the $x$ direction perpendicular to the direction of propagation. A similar elliptical elongation of the separatrix was found for a dipolar structures, where the function $f$ contained a small cubic part, independently by Masuda ${ }^{31}$ and Nycander ${ }^{32}$ by employing a perturbation theory, with the Lamb-type dipole as the zero-order solution. Also, we see that the structure has become more compact, the branches of the scatterplot inside the separatrix are closer than for the initial shielded dipole, indicating that the functional relationship between the vorticity and the streamfunction has changed significantly.

\section{B. Evolution and structure of the nonlinear dipole}

From Fig. 3 we get the impression that the nonlinear dipole constitutes a new stationary dipole vortex. To confirm this, we have isolated the nonlinear dipole, rescaled it to a higher resolution by a numerical interpolation, and used that as the initial conditions for a long-time integration of the Euler equations. In Fig. 4 we show the scatterplot of the a)

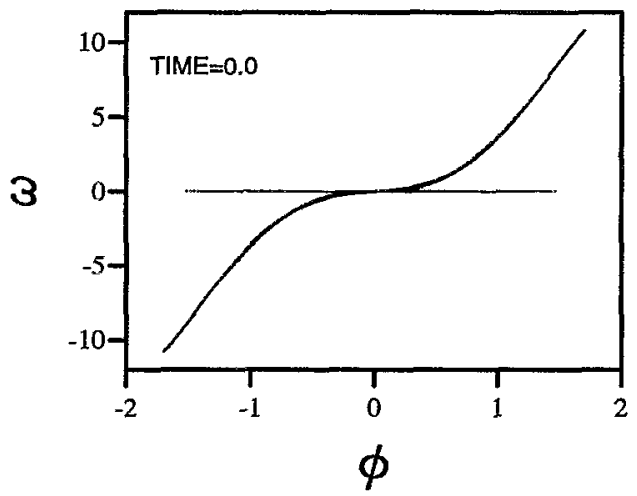

b)

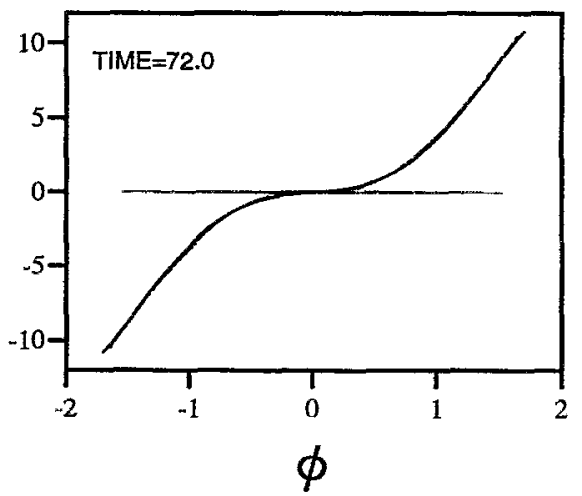

FIG. 4. The initial scatterplot (a) and the final scatterplot (b) where the temporal separation between the two corresponds to a propagation distance of 15 dipole diameters. Shown on both scatterplots are the least squares fit given by Eq. (20). The points and the curve from Eq. (20) are almost indistinguishable. 

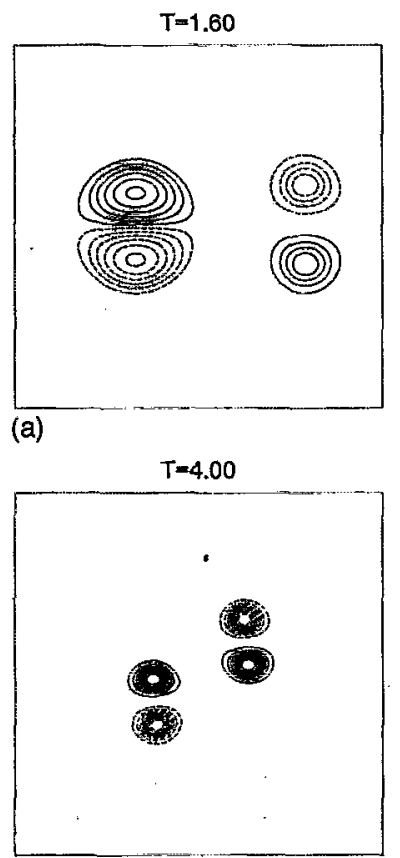

(b)
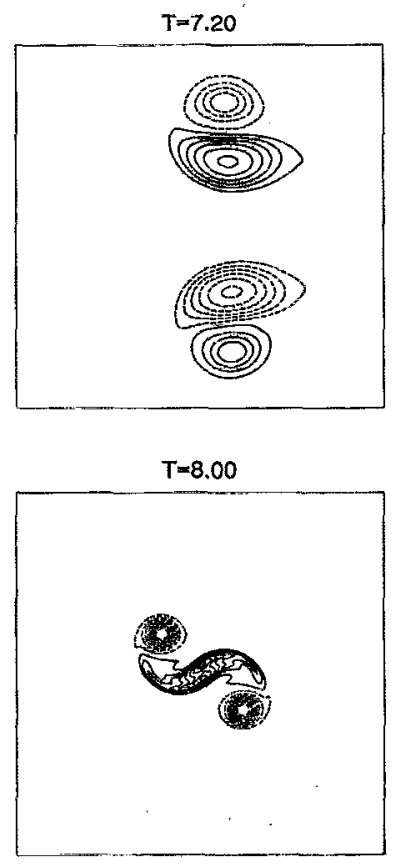

$T=12.80$
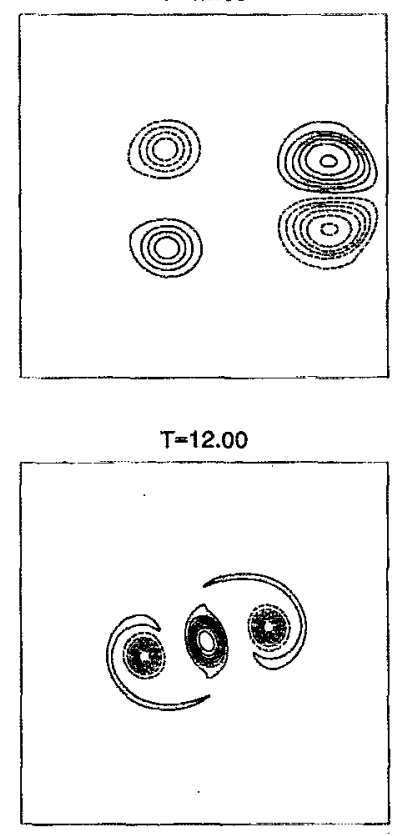

FIG. 5. Contour plot of the vorticity showing (a) a head-on collision between a product dipole and a Lamb dipole and (b) an off-axis collision between two product dipoles and the resulting creation of a tripole. In both cases the resolution is $N_{x}=256$ and $N_{y}=256$.

initial and final state of the nonlinear dipole. The final state corresponds to a time at which the nonlinear dipole has propagated more than 15 times its own width. Comparing the two scatterplots leads us to the conclusion that the reorganized nonlinear dipole is indeed a stationary solution to the Euler equations. Performing a least squares fit to the scatterplot we find an approximate functional relationship between the vorticity and the streamfunction given as

$$
\omega \cong 0.7 \phi+3.4 \phi^{3}-0.5 \phi^{5} \text {. }
$$

In Figs. 4(a) and 4(b) the functional form, Eq. (20), is shown by full lines.

As briefly discussed in the Introduction, by applying local maximum-entropy arguments it was shown ${ }^{17-20}$ that a natural state of the relaxed Navier-Stokes equations is a structure with a functional relationship given as

$$
\omega=A \sinh (c \phi) \cong A c \phi+\frac{A c^{3}}{6} \phi^{3}+\frac{A c^{5}}{120} \phi^{5}+\cdots,
$$

where $A$ and $c$ are constants. This has been verified in extensive numerical studies ${ }^{18.19}$ of the evolution of twodimensional flows in a double periodic domain using random initial conditions, with the restriction that the total circulation vanishes. This relationship has often been invoked to explain the observed nonlinear dipolar structures. It is clear that the dipole we have studied cannot be considered as a sinh solution due to the sign on the fifth-order term [Eq. (20)]. The sinh relationship is derived for initial vorticity distributions consisting of discrete values of the vorticity with the total vorticity being zero, and the negative and positive vorticity parts are conserved separately. Recently, Pasmanter ${ }^{22}$ consid- ered an initial random vorticity distribution with Poisson-like distribution and derived the following relationship for the most probable state:

$$
\omega \approx A \frac{\phi}{c-\phi^{2}}=\frac{A}{c} \phi+\frac{A}{c^{2}} \phi^{3}+\frac{A}{c^{3}} \phi^{5}+\cdots
$$

This relationship was found to fit the recent experimental results on dipoles formed by a turbulent jet in a stratified flow of Flor and van Heijst ${ }^{3}$ equally well as the sinh relationship. But as also noted by Pasmanter ${ }^{22}$ and Flor, ${ }^{3}$ a cubic polynomium would also provide a satisfactory fit, as could be expected at the relative low "amplitudes" where the fifthorder term is vanishing. Also, the form in Eq. (22) is different from the form obtained for the dipole studied here [Eq. (20)] due to the sign difference on the fifth-order term. Furthermore, it was pointed out by Robert and Sommeria ${ }^{21}$ that in the limit of a "dilute" initial vorticity distribution $f^{\prime}$ should have the largest value at the center of the vortices and no maxima should occur elsewhere. This is in agreement with the relationships in Eqs. (21) and (22). However, this does not agree with the relationship we have observed [Eq. (20)] for the nonlinear dipole investigated here, where $f^{\prime}(\phi)$ is maximum for $\phi=1.428$, with $f=7.935$. We find a tendency for a saturation of $f$, when $\omega$ approach its initial extremum values $\approx 10.5$ (of the shielded dipole). Note that the extremum of $\omega$ cannot exceed this initial value due to the conservation of vorticity in the Euler equations. This is in agreement with the general relationship found by Robert and Sommeria, ${ }^{21}$ when the initial condition consists of vortices with a distribution of strengths and the dilution approximation is not applied. A similar saturation effect has recently 
a)

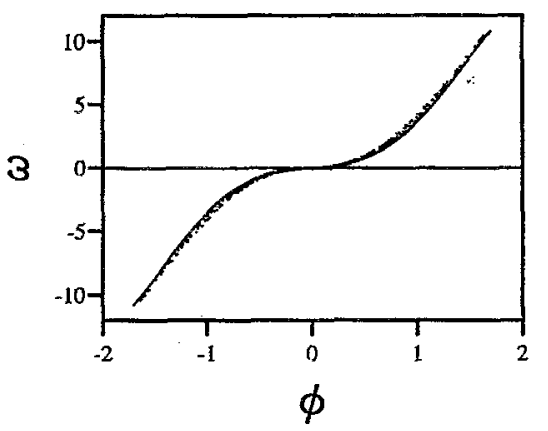

b)

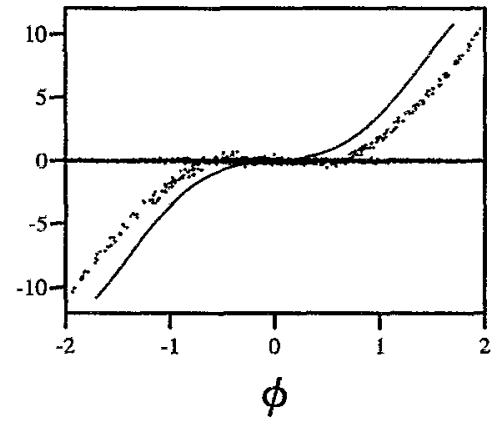

c)

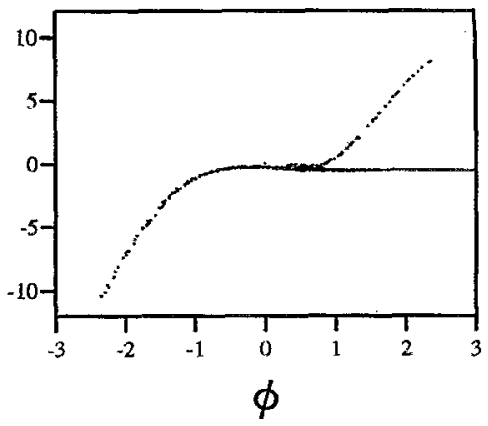

FIG. 6. Scatterplot of (a) the product dipole atter the collision with itself, (b) the product dipole after the collision with a Lamb dipole in Fig. 5(a). Full lines in the two plots are the least squares fits given by Eq. (20), and (c) of the tripolar vortex in Fig. 5(b) (here $\omega$ and $\phi$ are evaluated in a frame of reference corotating with the tripole ${ }^{16}$ ).

been observed in numerical investigations of the organization of a turbulent vortex street by Thess et al. ${ }^{33}$

\section{Collisions between dipoles}

In the previous subsection, we revealed that the nonlinear dipole was steadily propagating, i.e., it seems to be stable. In this subsection, we will study the full nonlinear dynamics of it by investigating its collision properties; see Figs. 5 and 6 . We initiate the study by performing a simulation of a head-on collision between two equivalent nonlinear dipoles. The two dipoles were found to approach each other, change partners and finally propagate in a direction exactly perpendicular to the initial direction of propagation in a way very similar to the collision of two identical Lamb dipoles. ${ }^{16}$ In Fig. 6(a) we show the scatterplot of one of the nonlinear dipoles after the collision together with the least squares fit from Eq. (20). It is clear that the dipole is stable to head-on collisions, as is the linear Lamb dipole.

The collision between a Lamb dipole and a nonlinear dipole is shown in Fig. 5(a). As there are several parameters that characterize the two different dipoles, there are many ways of setting up such an experiment. We have here chosen the parameters so that the two dipoles have approximately the same diameter and the same enstrophy. As the two cores are closer for the Lamb dipole, it will have a greater speed than that of our nonlinear dipole in this case. The simulation reveals a more complicated dynamics than in the previous case. We see the two dipoles exchange partners smoothly and as the two halfs of the Lamb dipole occupy a larger area they will convect the others on a circular trajectory. After half a turn they meet again, exchange partners, and after a reorganization both the Lamb dipole and the nonlinear dipole will reappear, with some scatter seen in the vorticity distribution. A closer examination of the reorganized nonlinear dipole reveals that the two centers are now further apart. As we are only interested in the nonlinear structure, we have cut away the reorganized Lamb dipole from Fig. 5(a) and continued the simulation with the nonlinear dipole alone. At the end of this simulation the dipole have relaxed to a stable structure with a scatterplot shown Fig. 6(b). Here, also the least squares fit from Eq. (20) is shown. We still have a nonlinear relation and the two branches have approximately the same form as before the collision, but the branches have been shifted from the origo. This shift is clearly related to the center separation.

It is well known that off-axis collisions of two Lamb dipoles, in some part of the parameter space, may cause the two dipoles to merge and finally form a stationary tripolar structure ${ }^{16}$ which has been studied extensively in the recent years. ${ }^{16,34}$ In Fig. 5(b) we show a simulation of an offaxis collision between two nonlinear dipoles, where the initial offaxis separation is $75 \%$ of the dipole width. Indeed, we see that the two positive partners of the dipoles merge and a tripolar structure is formed after the thin patches of positive vorticity wrapped around the negative vortices have died away. This structure then rotates steadily around a fixed center. The scatterplot of the tripolar vortex is shown in Fig. 6(c). We observe two branches, one of them corresponds to the positive central vortex and the other to the two negative satellite vortices. The amplitude of the positive and negative vortices are approximately equal and each of the branches have a functional relationship close to the form they come from, as described by Eq. (20). Also, we observe a flat part between the two branches indicating a relatively large separation between the central vortex and the two satellites. The tripolar structure found here has some similarities with the structure found from the off-axis collision of two Lamb dipoles, ${ }^{16}$ but it is even less compact and the functional relationship of the branches is somewhat different. Comparing the tripolar structure found in Fig. 5(b) with the structures found by Orlandi and van Heijst ${ }^{16}$ strengthens their statement that there does not seem to exist a "unique" tripolar structure. From further dipole collision studies we have found, that if the initial separation is less than approximately $1 / 3$ of the initial diameter, the two dipoles undergo a strong distortion and two asymmetric dipoles appear, leaving behind some shedded vorticity. We further observed that if the two dipoles àre placed more than $80 \%$ off axis they simply pass each other.

\section{Dissipative effects on the nonlinear dipole structủre}

The results of the forgoing sections are obtained for essentially inviscid flows. Here we examine the effects of the 

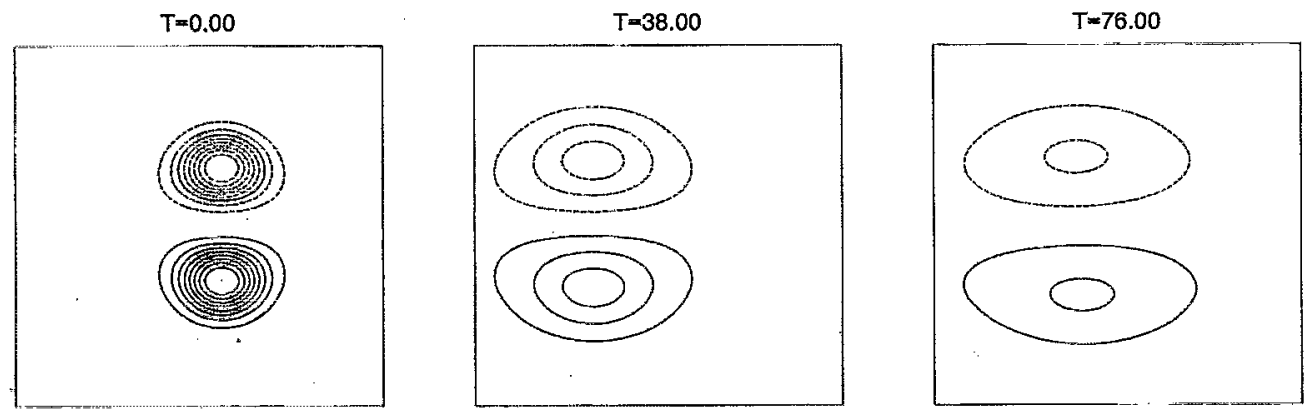

FIG. 7. Contour plot of the vorticity of the product dipole where a Newtonian viscosity term has been included in the simulation. The Reynold's number is Re 2000 and the spectral resolution is $N_{x}=256$ and $N_{y}=256$.

nonlinear dipole of a Newtonian viscosity, represented by a term $\nu \nabla^{2} \omega$ added on the right hand side of Eq. (1). Figure 7 shows a simulation with the nonlinear dipole as initial condition, where the kinematic viscosity term has been included. Defining a Reynold's number for the dipole as: $\operatorname{Re} \equiv U R / \nu$, where $U$ is the dipole speed and $R$ is the distance between the extrema of the two poles, we have $R e \approx 2000$. This number is comparable with $\mathrm{Re}$ for dipoles generated in rotating water tanks, see, e.g., Coutsias et al. ${ }^{6}$ At the end of the simulation the dipole has moved a distance of nine times its own size, the energy loss is $70 \%$ and the enstrophy loss is $86 \%$. The dipole has expanded uniformly in all directions and the amplitude and velocity have decreased. This evolution is different from the viscous decay of a Lamb dipole, which for this relative high Re was observed to almost keep its shape and only the amplitude and velocity were decreasing. Careful inspections, however, revealed the formation of an expanding tail lagging behind the dipole, which becomes more and more pronounced for decreasing Reynold's numbers. The same behavior was also found by Kida et al. ${ }^{35}$ who explained it by the enhanced viscosity effects at the separatrix of the Lamb dipole, which would smear out the strong gradients there with the result that the nonlinear term becomes nonzero and acts in such a way that a tail is shed off the structure. This behavior also appears to have been observed experimentally. ${ }^{3}$ Continuing the simulation even further than the last frame in Fig. 7, we found that the nonlinear dipole will continue to expand until it starts to interact with itself due to the periodic boundary conditions employed. The modifications of the dipole due to the viscosity is also visible in the scatterplot; see Fig. 8. The functional relationship between $\phi$ and $\omega$ has clearly lost the form given by Eq. (20), but we see that it still has a nonlinear form and it turns out that it fits very closely to a sinh function:

$$
\omega=0.85 \sinh (2.1 \phi) .
$$

It should be noted, however, at the low values of $\phi$ it is equally well fitted by a third-order polynomial: $\omega=1.79 \phi+1.31 \phi^{3}$. Thus this appears to be consistent with the results of Montgomery et al. ${ }^{18,19}$ who found that relationship for the ultimate vortex pairs appearing in freely decaying turbulence, as briefly discussed in Secs. I and III A. It may, however, be questioned if this vortex pair structure, filling the total periodic box, has any relation to freely moving dipoles emerging from localized forcing.

We should point out, however, that the sinh function will not be a final state in the general case. When starting the calculations with two vortices with Gaussian streamfunctions of opposite sign we found that the structure quickly reorganizes to a moving dipole with a weak nonlinear relationship between $\omega$ and $\phi$, as also found by Couder and Basdevant ${ }^{8}$ and McWilliams. ${ }^{15}$ However, under the action of Newtonian viscosity this relationship approached a linear relation, i.e., a Lamb dipole.

\section{CONCLUSIONS}

We revealed the existence of stationary solutions to the Euler equation with a localized streamfunction and nontrivial symmetry. The solution is explicitly found by a numerical two-dimensional shooting method. It appears as a dipolar structure with two cores of opposite vorticity that are shielded. The structure is found to be unstable by numerical solutions of the Euler equations with the dipolar structure as initial condition. However, a new strong, moving dipole emerges with a functional relationship between vorticity and streamfunction that is different from what has been considered previously. This structure has an elliptical separatrix elongated in the direction perpendicular to the direction of propagation. It is found to be stable, we have followed its

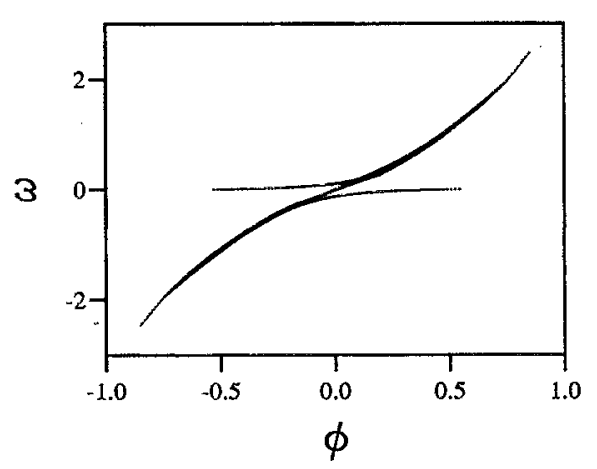

FIG. 8. Scatterplot of the product dipole from the last frame in Fig. 7. The full curve shows the fitted sinh dependence from Eq. (23). 
evolution over long times, where it has moved several times its own size (more than 25 times). During that time, the functional relationship between vorticity and streamfunction does not change. We should note that this nonlinear dipole is characterized by a monotonous functional relationship $\omega=f(\phi)$, which appears to be always the case for maximum entropy states. ${ }^{21}$ The initial shielded dipole has a nonmonotonous $f$, and its instability and reorganization may be interpreted as a tendency for the system to reach a state of higher entropy.

The nonlinear dipole is also found to be very robust and it has "collision characteristics" very similar to the Lamb dipole. It "survives" mutual head-on collisions. For off-axis collisions, a tripolar structure may evolve. Collisions between the nonlinear dipole and a Lâmb dipole show a more complex behavior, but the nonlinear dipole reappears with a slightly different form, the distance between the poles has increased.

A Newtonian viscosity will naturally damp the nonlinear dipole and its area is slightly increased, while the function $f$ was found to approach a sinh form. This, however, cannot be considered a general form, since we observed that a dipole originating from two vortices with Gaussian streamfunction of opposite sign would evolve into a linear function $f$. This issue will be discussed further in a forthcoming publication.

The detailed dipole structure depends strongly on the initial condition and to some extent also on the resolution. With a resolution of $N_{x}=256$ and $N_{y}=1024$ a slight shielding of the moving dipole was still attached, but also that structure behaved as the one we have treated in this paper. Finally, it seems that there is no "unique" dipolar structure. We conjecture that a wide class of dipolar structures emerging from localized initial conditions are possible, and they will all be well described by a relationship of the form $\omega=\alpha \phi+\beta \phi^{3}+\gamma \phi^{5}$, for small $\phi$ values this may be well fitted by either the sinh form or the form given in Eq. (22) or most naturally by a cubic relationship.

\section{ACKNOWLEDGMENTS}

One of the authors (JSH) was partly funded by UNIeC and partly by the National Science Academy which he gratefully acknowledges. The work of EGS and SKT was supported in part by ISF SOROS Foundation under Grant No. RCF000. Discussions with G. J. F. van Heijst, J. B. Flor, E. A. Coutsias, and B. Stenum on various aspects of the dynamics of dipolar vortices are gratefully acknowledged. We thank an anonymous referee for useful comments.

${ }^{1}$ G. J. F. van Heijst and J. B. Flor, "Dipole formations and collisions in a stratified flow," Nature 340, 212 (1989); "Laboratory experiments on dipole structures in a stratified fluid," in Mesoscale/Synoptic Coherent Structures in Geophysical Turbulence, edited by J. C. J. Nihoul and B. M. Jamart (Elsevier, Amsterdam, 1989), pp. 591-608.

${ }^{2}$ S. I. Voropayev, Ya. D. Afanasyev, and I. A. Filippov, "Horizontal jets and vortex dipoles in a stratified fiuid," J. Fluid Mech. 227, 543 (1991); "Twodimensional vortex-dipole interactions in a stratified fluid," 236, 665 (1992).

3J.-B. Flor, "Coherent vortex structures in stratified fluids," thesis, The Technical University of Eindhoven, The Netherlands, 1994; J.-B. Flor and G. J. F. van Heijst, "An experimental study of dipolar vortex structures in a stratified fluid," J. Fluid Mech. 279, 101 (1994).
${ }^{4}$ G. R. Flierl, M. E. Stern, and J. A. Whitehead, "The physical significance of modons: laboratory experiments and general integral constraints," Dyn. Atmos. Oceans 7, 233 (1983).

${ }^{5}$ R. C. Kloosterziel, G. F. Carnevale, and D. Philippe, "Propagation of barotropic dipoles over topography in a rotating tank," Dyn. Atmos. Oceans 19, 65 (1993).

${ }^{6}$ E. A. Coutsias, J. P. Lynov, A. H. Nielsen, M. Nielsen, J. Joul Rasmussen, and B. Stenum, "Vortex dipoles colliding with curved walls," in Further Directions of Nonlinear Dynamics in Physical and Biological Systems, edited by P. L. Christiansen, J. C. Eilbeck, and R. D. Parmentier (Plenum, New'York, 1993), pp. 51-54.

${ }^{7}$ J. M. Nguyen Duc and J. Sommeria, "Experimental characterization of steady two-dimensional vortex couples," J. Fluid Mech. 192, 175 (1988).

${ }^{8} \mathrm{Y}$. Couder and C. Basdevant, "Experimental and numerical study of vortex couples in two-dimensional flows," J. Fluid Mech. 173, 225 (1986).

${ }^{9} \mathrm{O}$. U. Velasco Fuentes and G. J. F. van Heijst, "Experimental study of dipolar vortices on a topographic $\beta$-plane," J. Fluid Mech. 259, 79 (1994).

${ }^{10}$ M. V. Nezlin and E. N. Snezhkin, Rossby Vortices, Spiral Structures, Solitons (Springer-Verlag, Berlin, Heidelberg, 1993).

${ }^{11}$ R. C. Kloosterziel and G. J. F. van Heijst, "An experimental study of unstable barotropic vortices in a rotating fluid," J. Fluid Mech. 223, 1 (1991).

${ }^{12}$ B. Legras, P. Santangelo, and R. Benzi, "High-resolution numerical experiments for forced two-dimensional turbulence," Europhys. Lett. 3, 37 (1988).

${ }^{13}$ H. Lamb, Hydrodynamics (Dover, New York, 1932).

${ }^{14} \mathrm{~V}$. V. Meleshko and G. J. F. van Heijst, "On Chaplygin's investigations of two-dimensional vortex structures in an inviscid fluid," J. Fluid Mech. 272, 157 (1994)

${ }^{15}$ J. C. McWilliams, "Interaction of isolated vortices: II Modon generation by monopole collision," Geophys. Astrophys. Fluid Dyn. 24, 1 (1983).

${ }^{16}$ P. Orlandi and G. J. F. van Heijst, "Numerical simulation of tripolar vortices in 2D flow," Fluid Dyn. Res. 9, 179 (1992).

${ }^{17} \mathrm{G}$. Joyce and D. Montgomery, "Negative temperature states for the twodimensional guiding-center plasma," J. Plasma Phys. 10, 107 (1973); D. C. Montgomery and G. Joyce, "Statistical mechanics of "negative temperature' states," Phys. Fluids 17, 1139 (1973).

${ }^{18}$ D. Montgomery, W. H. Matthaeus, W. T. Stribling, D. Martinez, and S. Oughton, "Relaxation in two dimensions and the 'sinh-Poisson' equation," Phys. Fluids A 4, 3 (1992).

${ }^{19} \mathrm{D}$. Montgomery, X. Shan, and W. H. Matthaeus, "Navier-Stokes relaxation to sinh-Poisson states at finite Reynolds numbers," Phys. Fluids A 5, 2207 (1993).

${ }^{20}$ R. A. Smith, "Maximization of vortex entrophy as an organizing principle in intermittent, decaying, two-dimensional turbulence," Phys. Rev. A 43, 1126 (1991).

${ }^{21} R$. Robert and J: Sommeria, "Statistical equilibrium states for twodimensional flows," J. Fluid Mech. 229, 291 (1991).

${ }^{22} R$. A. Pasmanter, "On long-lived vortices in 2-D viscous flows, most probable states of inviscid 2-D flows and a soliton equation," Phys. Fluids 6, 1236 (1994).

${ }^{23}$ M. J. Ablowitz and H. Segur, Solitons and the Inverse Scattering Transform (SIAM, Philadelphia, 1981).

${ }^{24}$ R. Chiao, E. Garmire, and C. Townes, "Self-trapping of optical beams," Phys. Rev. Lett. 13, 479 (1964).

${ }^{25} \mathrm{Z}$. Jankauskas, "Radial field distributions in self-trapping light beams," Izv. Vuzov Radiophys. 9, 412 (1966).

${ }^{26}$ G. L. Alfimov, V. M. Eleonsky, N. E. Kulagin, L. P. Lerman, and V. P. Silin, "On existence of nontrivial solutions to the equation $\Delta u-u+u^{3}=0$," Physica D 44, 168 (1990).

${ }^{27}$ The localized dipolar solution of Eq. (5) were first obtained by two of the authors (EGS and SKT) in the context of the nonlinear Schrödinger equation, describing $f$ in two parallel waveguide channels in a nonlinear optical medium; here $\phi$ designates the envelope of the optical field. In that context we would expect these waveguides to be unstable for twodimensional perturbations, which may ultimate lead to a collapse of the waveguides.

${ }^{28}$ P. R. Gent and J. C. McWilliams, "The instability of barotropic circular vortices," Geophys. Astrophys. Fluid Dyn. 35, 209 (1986).

${ }^{29}$ P. Orlandi, R. Verzicco, and G. J. F. van Heijst, "Stability of shielded vortex dipoles," in Modelling of Oceanic Vortices, edited by G. J. F. van Heijst (North Holland, Amsterdam, 1994).

${ }^{30}$ E. A. Coutsias, F. R. Hansen, T. Huld, G. Knorr, and J. P. Lynov, "Spectral 
methods in numerical plasma simulations," Phys. Scr. 40, 270 (1989).

${ }^{31}$ A. Masuda, "A skewed eddy of Batchelor-Modon type," J. Oceanogr. Soc. Jpn. 44, 189 (1988).

${ }^{32} \mathrm{~J}$. Nycander, "New stationary vortex solutions of the Hasegawa-Mima equation." J. Plasma Phys. 39, 413 (1988).

${ }^{33} \mathrm{~A}$. Thess, J. Sommeria, and B. Juttner, "Internal organization of a two- dimensional turbulent vortex street," Phys. Fluids 6, 2417 (1994).

${ }^{34}$ L. M. Polvani and X. J. Carton, "The tripole: A new coherent vortex structure of incompressible two-dimensional flows," Geophys. Astrophys. Fluid Dyn. 51, 87 (1990).

${ }^{35} \mathrm{~S}$. Kida, M. Takaoka, and F. Hussian, "Formation of head-tail structure in a two-dimensional uniform straining flow," Phys. Fluids A 3, 2688 (1991). 